Modern Physics Letters A

Vol. 35, No. 11 (2020) 2050079 (14 pages)

(C) The Author(s)

DOI: $10.1142 /$ S0217732320500790

\title{
Experimental hint for gravitational CP violation
}

\author{
Vahagn Gharibyan \\ Deutsches Elektronen-Synchrotron DESY, D-22603 Hamburg, Germany \\ vahagn.gharibyan@desy.de
}

Received 23 June 2019

Revised 2 September 2019

Accepted 4 December 2019

Published 3 January 2020

\begin{abstract}
An equality of particle and antiparticle gravitational interactions holds in general relativity and is supported by indirect observations. Gravity dependence on rotation or spin direction is experimentally constrained for non-relativistic matter. Here, a method based on high-energy Compton scattering is developed to measure the gravitational interaction of accelerated charged particles. Within that formalism, the Compton spectra measured at HERA rule out the speculated anti-gravity possibility for antimatter at a confidence level close to $100 \%$. The same data, however, imply a gravitational CP violation around $13 \mathrm{GeV}$ energies, by a maximal amount of $(9 \pm 2) \cdot 10^{-12}$ for the charge and $(13 \pm 3) \cdot 10^{-12}$ for the space parity. The detected asymmetry hints for a stronger gravitational coupling to left helicity electrons relative to right helicity positrons.
\end{abstract}

Keywords: CP invariance; experimental test of gravitational theories; relativistic electron and positron beams in accelerators.

PACS Nos.: 11.30.Er, 41.75.Ht, 42.60.-v

\section{Introduction}

Extreme weakness of gravitation makes it the least experimentally investigated fundamental interaction at sub-atomic scales, with elementary particles. Any test with a single carrier of the gravitational force, hypothetical graviton, is unfeasible whereas in the electroweak or strong interactions experimental results with a photon, W, Z boson or a gluon are readily available at sufficiently high energies. The weak gravity combined with a rarity and vulnerability of antiparticles drives any attempt of testing the antimatter gravitation to its technical limits. Einstein's general relativity, $\frac{1}{1}$ the currently accepted theory of gravitation, does not distinguish

This is an Open Access article published by World Scientific Publishing Company. It is distributed under the terms of the Creative Commons Attribution 4.0 (CC BY) License which permits use, distribution and reproduction in any medium, provided the original work is properly cited. 
between particles and antiparticles or their properties such as spin or helicity. The general relativity is based on universality of gravity and equivalence principle, and, there is no alternative theory available to predict or describe violations of these principles in a consistent way. Departures from perfect spin or particle-antiparticle symmetry are allowed in some quantum gravitation scenarios. ${ }^{2}$ Hence, observations of antiparticle gravitation could serve as an experimental input for quantum

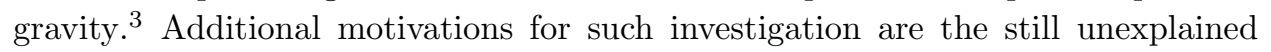
matter-dominant universe ${ }^{4}$ and the connection of antimatter's possible anti-gravity ${ }^{5}$ to the accelerated expansion of the universe. ${ }^{6}$ One can also think about a possible particle-antiparticle gravitational asymmetry and helicity dependence from an analogy to electroweak interactions, where a photon's massive partners, $\mathrm{W}$ and Z bosons, are considered responsible for space and charge parity violations. ${ }^{7}$ Thus, possible massive or lower spin gravitons could introduce similar violations 8 that may remain hidden at low energies and will become detectable at high energies.

Another possibility to incorporate asymmetric space or charge gravitational interactions is a modification of vacuum properties described within the Standard Model Extension (SME) ${ }^{9}$ by Lorentz violating terms in Lagrangian. Such actionbased approach is further developed for anomalous antimatter gravity in Ref. 10 .

Indirect observations of matter-antimatter gravitational asymmetry involve nuclei with different content of quark-antiquarks in the equivalence principle Eötvös type experiments. ${ }^{11,12}$ Using CPT conservation, the observed stringent limits for the equivalence principle violating matter could be expanded to a limit below $10^{-7}$ for the matter-antimatter low-energy gravitational asymmetry. ${ }^{13}$ Hughes and Holzscheiter have set limits on the matter-antimatter gravitational differencies from an equality of particle-antiparticle cyclotron frequencies. ${ }^{14}$ As the source of gravitation, however, they have used the potential of the local supercluster while for the Earth's potential, the derived restrictions are completely lifted. Technical difficulties for charged antiparticle's gravitational coupling's direct measurements turned physicists' attention to neutral antimatter tests ${ }^{15-17}$ which may deliver conclusive results soon. The ongoing experiments, however, are still at low-energy, and massive gravitons' interactions may remain unseen.

Spin-dependent gravitation (for a review, see Ref. 18) motivated by Lorentz violation,, 19 torsion gravity, $\underset{20}{ }$ exchange of pseudoscalar bosons 21 or few other hypothesis has been constrained by low-energy spin-polarized experiments, such as the test with polarized torsion pendulum. ${ }^{22}$ Here also, the high-energy could reveal gravitation's preference for a helicity which is hidden at low energies.

In this paper, I will demonstrate an extreme sensitivity of a high-energy process - laser Compton scattering - to an antiparticle's hypothetical anti-gravity and gravitational charge and space parity violation. Next, applying the developed formalism to the existing data of the HERA Compton polarimeter, I will compare the $\gamma$-spectra generated by electrons and positrons to measure the charge and spin asymmetry for their gravitational interaction. Systematic effects and prospects for other tests will be discussed at the end. 


\section{Refraction in Gravitational Field}

In an earlier publication, high-energy Compton scattering sensitivity has been shown to a Planck scale dispersive and birefringent vacuum model. ${ }^{23}$ Subsequently, I applied the same formalism to the Earth's gravity assuming the real gravitational field's induced refractivity only for the Compton photons. ${ }^{24}$ The refraction, however, also affects the leptons involved in the scattering ${ }^{25}$ in agreement with the equivalence principle. This makes the Ref. 24 conclusions on the general relativity violation invalid. ${ }^{\mathrm{a}}$

Here, I follow the formalism developed by Evans et al. ${ }^{25}$ to find a massive particle's energy-momentum or refraction relation in a static and isotropic gravitational field described by the Schwarzschild metric. Combining Eqs. (3) and (30) from Ref. 25 , for a particle at a distance $R$ from mass $M$, in a weak gravitational field $G M / R \ll c^{2}$, one can derive a refraction relation

$$
c \frac{P}{\mathcal{E}}=\frac{v}{c}+\frac{2 G M}{c^{2} R},
$$

where $G$ is the gravitational constant, $c$ is the speed of light and $\mathcal{E}, P$ and $v$ are energy-momentum and velocity of the particle. This relation is also valid for massless particles: at $v=c$, it describes the photon refraction in a gravitational field in a form derived by many authors; see Ref. 26 and references therein, or, for a more recent reference, see Ref. 27. The second term in Eq. (1) is a scaled gravitational potential $U(G, M, R)=\overline{-G} M / R$ of the gravitating mass. This term can be dropped from Eq. (1) unless there is a change in the potential

$$
\Delta U=U \frac{\Delta G}{G}+U \frac{\Delta M}{M}-U \frac{\Delta R}{R} .
$$

Indeed, at $\Delta U=0$, the physical processes (involving one or more particles) are not altered by the extra gravitational term in Eq. (1) since the potential $U$ cancels in energy-momentum conservation. Situation changes with $\Delta U \neq 0$. Thus, for $\Delta R \neq 0$ case, differentiated Eq. (1) describes particle's gravitational energy change (frequency redshift/blueshift for $v=c$ ) or deflection - using the principle of least action. While these are classical gravity processes validated by multiple measurements, the first term in Eq. (2), $\Delta U_{G}=U \Delta G / G$, violates equivalence principle and has intensively been constrained by pendulum null experiments with different materials - all for the non-relativistic $(c=\infty)$ case (see Ref. 11 and references therein). Thus, Eq. (1) follows from general relativity in a weak field (metric is replaced by gravitational potential) and describes relativistic gravitational effects in accord with the equivalence principle.

To allow departure from the equivalence principle, let us retain the interaction strength $G$ for matter particles and use a different strength $G_{p}$ for antimatter 
leptons to write Eq. (1) for positrons in the following form:

$$
c \frac{P}{\mathcal{E}}=\frac{v}{c}-2 \frac{U}{c^{2}}\left(1+\frac{\Delta G_{p}}{G}\right),
$$

with $\Delta G_{p}=G_{p}-G$. For an anti-gravitating positron $G_{p}=-G$.

In a similar manner, within the spin affected gravitation, we can assign an interaction constant $G_{-}$to particles possessing a negative(left) helicity and modify Eq. (1) to include a helicity-dependent term $\Delta G_{-}=G_{-}-G$

$$
c \frac{P}{\mathcal{E}}=\frac{v}{c}-2 \frac{U}{c^{2}}\left(1+\frac{\Delta G_{-}}{G}\right) .
$$

Positive(right) helicity particles' coupling constant $G_{+}$is assumed to provide an average $\left(G_{-}+G_{+}\right) / 2=G$ for unpolarized or zero helicity case, so that $\Delta G_{-}=$ $-\Delta G_{+}$.

Anomalous dispersion or refraction relations similar to Eq. (3) or (4) are often applied for vacuum, within the context of action-based Lorentz violation. ${ }^{28,29}$ Such models have an advantage to access modified dynamic properties (cross-sections) of considered processes though stringent observational and experimental limits, in particular from a Compton scattering test, $\frac{32}{2}$ exist for Lorentz violating vacuum. $\frac{30}{}$

Here, we limit ourselves exceptionally with kinematics in a real gravitational field to investigate how the hypothetical refractions by Eqs. (3) and (4) are affecting the high-energy laser Compton process.

Similar to Eq. (3), assumption has recently been considered in hypothetical vacuum Cherenkov radiation formalism to constrain charge parity violation in gravitational field. 31

\section{The Compton Process Affected by Gravity}

Using energy-momentum conservation with Eqs. (1) and (3), when in the Earth's gravitational field a photon scatters off a positron with energy $\mathcal{E}$, the Compton scattering kinematics is given by (from here on natural units are assumed)

$$
\mathcal{E} x-\omega\left(1+x+\gamma^{2} \theta^{2}\right)+4 \omega\left(1-\frac{\omega}{\mathcal{E}}\right) \gamma^{2} U \frac{\Delta G_{p}}{G}=0,
$$

where $x=4 \gamma \omega_{0} \sin ^{2}\left(\theta_{0} / 2\right) / m$, with $m$ and $\gamma=\mathcal{E} / m$ being the mass and Lorentz factor of the initial positron, respectively. The initial photon's energy and angle are denoted by $\omega_{0}$ and $\theta_{0}$, while the refraction of Eq. (1) is in effect for the scattered photon with energy $\omega$ and angle $\theta$; the angles are defined relative to the initial positron. This kinematic expression is derived for weak gravity and high energies, i.e. the $\mathcal{O}\left(U^{2}\right), \mathcal{O}\left(\theta^{3}\right)$, and $\mathcal{O}\left(\gamma^{-3}\right)$ terms are neglected. In this approximation, the refraction effect of Eq. (1) for the initial laser photon is negligible. For the initial and scattered positrons the refraction of Eq. (3) is applied. To determine the outgoing photon's maximal energy, Eq. (5) is solved for $\omega$ at $\theta=0$ with the following result:

$$
\omega_{\max }=\mathcal{E} \frac{b+q-\sqrt{b^{2}+q(q-2 b+4)}}{2 q},
$$




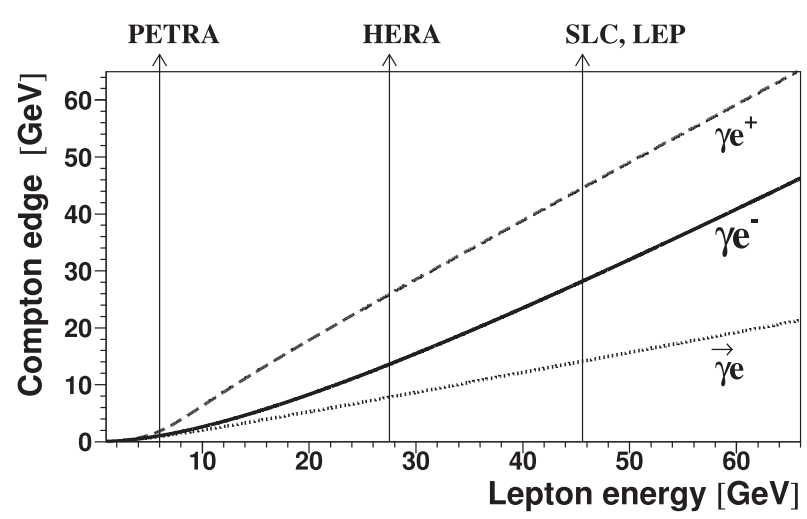

Fig. 1. The maximal energy of Compton scattered photons (Compton edge) and its dependence on the initial lepton energy for a head-on collision with $532 \mathrm{~nm}$ laser light, in the Earth's gravitational field. The solid line is for matter gravity $\left(e^{-}\right.$, electron, $G$ ) while the dashed line shows antimatter anti-gravity case $\left(e^{+}\right.$, positron, $\left.-G\right)$. The dotted line corresponds to gravitationally sterile right (positive helicity) Compton photons $\left(\vec{\gamma}, G_{+}=0\right)$. Names of $e^{+} e^{-}$accelerators are printed at the upper part.

where $b=1+x$ and $q=-2 \gamma^{2} U \Delta G_{p} / G$. Thus, in high-energy Compton scattering, the small factor $U \Delta G_{p} / G$ is amplified by $\gamma^{2}$, allowing one to measure it by detecting the extreme energy of the scattered photons $\omega_{\max }$, or positrons $\mathcal{E}-\omega_{\max }$ (Compton edge).

In order to estimate the method's sensitivity, I calculate the Compton edge from Eq. (6) for an incident photon energy $2.32 \mathrm{eV}$ (the widely popular green laser) in the Earth's gravitational field $\left(U=-G M_{\oplus} / R_{\oplus}=-6.95 \times 10^{-10}\right)$, at different energies of the accelerator leptons. The resulting dependencies for a matter (electron) gravity and antimatter (positron) anti-gravity are presented in Fig. 1. The plot shows considerable sensitivity, which grows toward high energies in a range available to accelerating laboratories. For handling measurement's systematic errors, from an experimental point of view, it is more precise to measure a relative asymmetry rather than absolute Compton edge energy. Therefore, we form an asymmetry of Compton edges measured on positrons $\left(\omega_{\max }^{p}\right)$ and electrons $\left(\omega_{\max }^{e}\right)$

$$
A=\frac{\omega_{\max }^{p}-\omega_{\max }^{e}}{\omega_{\max }^{p}+\omega_{\max }^{e}},
$$

and use Eq. (5) to find the charge parity gravitational violation magnitude

$$
\Delta U_{G p} \equiv U \frac{\Delta G_{p}}{G}=-\frac{1}{2 \gamma^{2}} \cdot \frac{A(1-A)(1+x)^{2}}{(1+A)(2 A x+A-1)} .
$$

For evaluating a sensitivity of the laser Compton process to the spin-dependent gravitation, let us investigate left helicity photon scattering off unpolarized (zero mean helicity) leptons' beam. According to helicity conservation, the scattered gamma-quantum will retain the helicity of the initial photon at Compton edge 


\section{Gharibyan}

energies. ${ }^{33}$ Hence, we apply the helicity modified refraction relation of Eq. (4) to the initial and final photons, reserving the refraction of Eq. (1) for the initial lepton. After scattering, the leptons become longitudinally polarized acquiring $P_{l}=x(2+x) /\left(1+(1+x)^{2}\right)$ portion of the initial photon circular polarization. ${ }^{34,35}$ Therefor, for the scattered lepton, the term $\Delta G_{-} / G$ in energy-momentum relation (4) must be scaled by a factor $-P_{l} / 2$, where the $-1 / 2$ coefficient counts for half spin and positive helicity of the lepton. This modifications will bring Eqs. (6) and (8) for the charge parity case to

$$
\omega_{\max }^{-}=\mathcal{E} \frac{\sqrt{b^{2}+4 u\left(u+x+P_{l}-1\right)}-b+2 u\left(1+P_{l}\right)}{2 u\left(2+P_{l}\right)},
$$

and, with a measured energy asymmetry $A_{-}=\left(\omega_{\max }-\omega_{\max }^{0}\right) /\left(\omega_{\max }^{-}+\omega_{\max }^{0}\right)$, to

$$
\Delta U_{G-}=\frac{1}{2 \gamma^{2}} \cdot \frac{2 A_{-}\left(1-A_{-}\right)\left(x^{2}+2 x+2\right)(1+x)^{2}}{A_{-}^{2} x_{1}+A_{-} x_{2}-\left(2 x^{2}+3 x+2\right)},
$$

for the space parity case. In the above equations, the upper index $(-, 0)$ stands for the scattered photon's average helicity and the following assignments are made: $u=-2 \gamma^{2} U \Delta G_{-} / G, \Delta U_{G-}=U \Delta G_{-} / G, x_{1}=8 x^{3}+22 x^{2}+21 x+6$, $x_{2}=4\left(x^{3}+x^{2}-0.5 x-1\right)$. Energy dependence of Eq. (9), if the Earth's gravity attracts only left helicity particles, is plotted in Fig. 1. From the plot and formulas, we can conclude that Compton process sensitivity to the gravitational space parity violation is sufficient for introducing a Compton edge sizable shift in a scenario of the helicity-dependent gravitational field.

For estimating the sensitivity in Fig. 1, the Earth's gravitational potential $6.95 \cdot 10^{-10}$ is used. There is though a much more greater gravitational field at the Earth's surface, the largest, local Supercluster's potential ${ }^{14}$ with a magnitude $3 \cdot 10^{-5}$. For gravitational effects induced by the third term proportional to $\Delta R / R$ in Eq. (2), the Earth's potential prevails (due to large $1 / R$ factor) while in our case, for the first term in Eq. (2) with $\Delta G / G$, the local Supercluster potential contribution is dominant. Exact potential selection, however, is important only when separating the $\Delta G / G$ contribution from the $\Delta U$ factor. The Compton scattering could measure only the factors $\Delta U_{G p}$ or $\Delta U_{G-}$.

Besides the gravitation, other interactions also may alter energy-momentum relation. ${ }^{36,37}$ For the Compton process, a background electromagnetic field is the main competitor to gravity, and, since electric fields are usually shielded by conductive beam pipes, let us estimate influence of magnetic fields. According to Eq. (1.1) from Ref. 36, the maximal impact to energy-momentum relation for photons in a magnetic field $B$ is presented by

$$
\frac{P}{\mathcal{E}}=1-\frac{14}{45} \frac{\alpha^{2}}{m^{4}} B^{2}
$$

where $\alpha$ is the fine-structure constant. Hence, refractivity created by a 4 T superconducting magnet is about $8 \cdot 10^{-21}$ and that of Earth's magnetic field is $10^{-25}$. These 
tiny effects are still experimentally unreachable, and, compared to the gravitational potential magnitude (either the Supercluster's $\sim 10^{-5}$ or the Earth's $\sim 10^{-9}$ ) in Eq. (1), are completely negligible.

\section{Experimental Results}

The high-energy accelerators where laser Compton facilities have been operated for years, are listed on the upper energy scale of Fig. 1. As can be seen from the plot, $6 \mathrm{GeV}$ storage rings have low sensitivity while the higher energy colliders (HERA, SLC, LEP) have a great potential for detecting gravity related energy shifts. This is true for the HERA and SLC Compton polarimeters but not for the LEP polarimeter, which has generated and registered many photons per machine pulse. $\frac{38}{} \mathrm{In}$ this multi-photon regime, any shift of the Compton edge is convoluted with the laserelectron luminosity and cannot be disentangled and measured separately. Unlike the LEP, the SLC polarimeter operated in multi-electron mode and analyzed the energies of interacted leptons using a magnetic spectrometer. ${ }^{39}$ However, at SLC only the electron beam was polarized, and positron data are missing. Hence, we turn to HERA, which has recorded Compton measurements for both the electrons and the positrons. At the HERA transverse polarimeter, Compton photons are registered by a calorimeter in single particle counting mode. A recorded Compton spectrum produced by $514.5 \mathrm{~nm}$ laser scattering on $26.5 \mathrm{GeV}$ electrons, from Ref. 40 is shown in Fig. 2 superimposed on a background Bremsstrahlung distribution. In contrast to Compton scattering, in the Bremsstrahlung process, the momentum transfer is not fixed, and any small refractive effect is smeared out and becomes negligible. $\stackrel{41}{ }$ Hence, following the analysis in Ref. 41, I calibrate the energy scale according to the maximal Bremsstrahlung energy which is found by fitting a convolution of parent

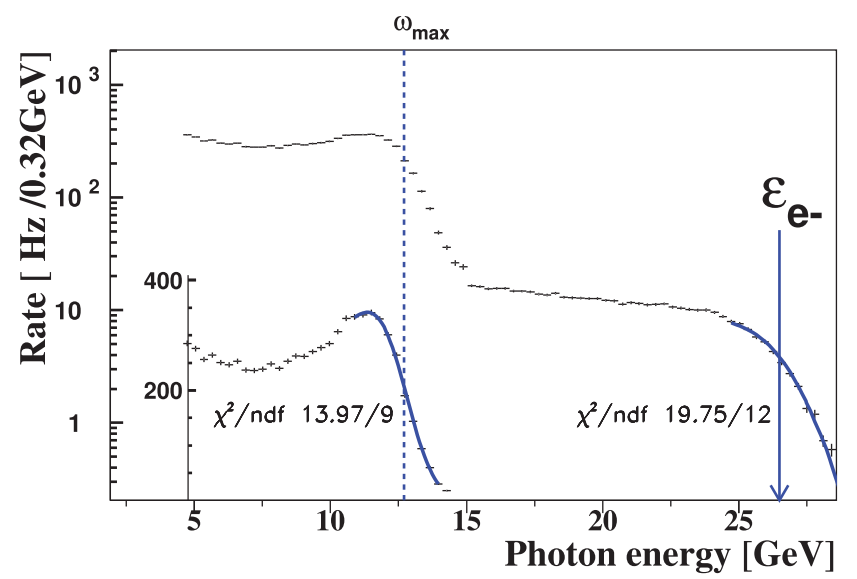

Fig. 2. HERA polarimeter Compton $\gamma$-spectrum produced by laser scattering on $26.5 \mathrm{GeV}$ electrons, on top of background Bremsstrahlung with fit results. The inset displays the background subtracted Compton spectrum. Vertical lines show measured values of the Compton $\left(\omega_{\max }\right)$ and Bremsstrahlung $\left(\mathcal{E}_{e-}\right)$ maximal energies. 
energy distribution $d \Sigma / d \omega$ with the detector response Gaussian function,

$$
F\left(E_{\gamma}\right)=N \int_{0}^{E_{m}} \frac{d \Sigma}{d \omega} \frac{1}{\sqrt{\omega}} \exp \left(\frac{-\left(\omega-E_{\gamma}\right)^{2}}{2 \sigma_{0}^{2} \omega}\right) d \omega
$$

to the Bremsstrahlung spectrum. $\sigma_{0}$ and $E_{\gamma}$ in the fitting function denotes the calorimeter resolution and detected photon's energy, respectively, while the normalizing factor $N$ and maximal energy $E_{m}$ are free fitting parameters. The fit is performed using MINUIT $\stackrel{42}{2}$ and the integral (12) is calculated numerically applying the Gaussian quadrature algorithm. The same fitting function with the Bremsstrahlung parent distribution replaced by the Compton scattering differential cross-section $d \Sigma_{C} / d \omega$ is applied to the background subtracted spectrum to find the Compton edge at $\omega_{\max }^{e 0}=12.70 \pm 0.02 \mathrm{GeV}$. Here, the upper indexes denote (scattered) lepton type, helicity and the 0 stands for a spectrum summed over the laser left and right helicities. The fit results together with fit quality estimates are shown in Fig. 2. The absolute value of the Compton edge is calculated from three measurements Bremsstrahlung and Compton edges $B_{\max }$ and $C_{\max }$ in ADC units, and electron beam energy $E_{\text {beam }}$ in $\mathrm{GeV}$ - by a formula $\omega_{\max }=E_{\text {beam }} C_{\max } / B_{\max }$. More details about the analysis and experimental setup can be found in Ref. 42 .

The same analysis procedure is applied to a HERA polarimeter Compton spectrum that was generated with left helicity photons scattered on $27.5 \mathrm{GeV}$ positrons and has been reproduced in Fig. 8 of Ref. 43. As mentioned above, left helicity laser photons are generating the same (negative) helicity scattered positrons at the Compton edge $\left(\omega_{\max }^{p-}\right)$. The resulting plots with fit quality outcomes are displayed in Fig. 3. Comparing the obtained Compton edge $\omega_{\max }^{p-}=13.80 \pm 0.02 \mathrm{GeV}$ with the photons' maximal energy for the anti-gravitating positrons $25.9 \mathrm{GeV}$, derived from Eq. (6), one can conclude without any advanced systematic error analysis

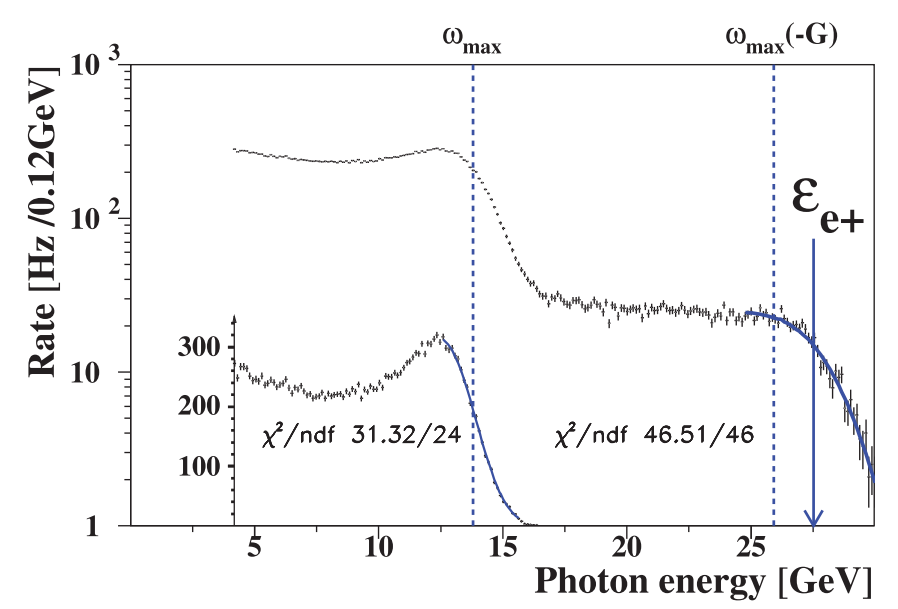

Fig. 3. A similar plot to Fig. 2 for positrons with energy $27.5 \mathrm{GeV}$. The Compton edge energy for anti-gravitating positrons is indicated by a vertical line $\omega_{\max }(-G)$. 
that anti-gravity for the positrons is ruled out. We can estimate accuracy of this statement by assuming an absolute energy calibration scale of $0.14 \mathrm{GeV}$ as it is calculated in Ref. 41. Thus, antimatter's anti-gravity is excluded within more than $85 \sigma$ confidence interval.

Using only the quoted statistical errors for the $\omega_{\max }^{e 0}, \omega_{\max }^{p-}$, we obtain a positron-electron and negative-zero helicity Compton edge asymmetry $A_{e p 0-}=$ $0.01297 \pm 0.0013$. In order to account for the different energies of accelerated electrons and positrons 26.5 and $27.5 \mathrm{GeV}$, the measured maximal energies in the asymmetry calculation have been normalized to 13.10 and $13.87 \mathrm{GeV}$ for the electrons and positrons, respectively. These are the expected Compton edge values from Eqs. (6) or (9) in the absence of gravitational anomaly, at $\Delta G_{p}=0$ or $\Delta G_{-}=0$, respectively.

Since the spectra for electrons and positrons are detected with the same experimental setup, i.e. with the same laser, geometry and detector, both measurements will experience the same systematic influences that will cancel out or reduce greatly in the asymmetry of Eq. (7). Hence, we omit systematic corrections with associated errors applied to polarization or energy measurements (as described in the Refs. 40, 41, 43 and 44), in order to analyze instrumental errors specific to the detected asymmetry which we rewrite in a more convenient form:

$$
A_{e p 0-}=\frac{\eta_{p} x_{e}\left(1+x_{p}\right)-\eta_{e} x_{p}\left(1+x_{e}\right)}{\eta_{p} x_{e}\left(1+x_{p}\right)+\eta_{e} x_{p}\left(1+x_{e}\right)},
$$

where $\eta=C_{\max } / B_{\max }$ is a ratio of the measured Compton and Bremsstrahlung edges, $x$ is the kinematic factor with indexes $e$ and $p$ denoting the measurement with electrons and positrons, respectively.

Uncertainty of the factor $x_{e, p}=4 \mathcal{E}_{e, p} \omega_{0} \sin ^{2}\left(\theta_{0} / 2\right) / m^{2}$ is dominated by the lepton beam energy spread while contribution of the other constituents is negligible: $\sigma\left(\omega_{0}\right) / \omega_{0} \approx 10^{-5}, \sigma(m) / m \approx 3 \cdot 10^{-7}, \Delta\left(\theta_{0}\right) \approx 2 \operatorname{mrad} \Rightarrow \Delta \sin ^{2}\left(\theta_{0} / 2\right) \approx 3 \cdot 10^{-6}$. Therefore, HERA leptons' energy spread $\sigma(\mathcal{E}) / \mathcal{E} \approx 10^{-3}$ will introduce a systematic uncertainty $7.9 \cdot 10^{-4}$ to the measured asymmetry. Since the electron and positron measurements are considerably separated in time, one of the important potential sources for a false asymmetry could be detector aging and degraded performance. This assumption could be checked by measuring the calorimeter resolution from the spectra edges' slopes - the same energy resolution $\sigma_{0}=0.24$ for both spectra indicates the same, non-degraded, response of the calorimeter.

A major energy correction factor, detector nonlinearity, applied to each measured Compton edge will largely cancel in the asymmetry. However, assuming a possible change of detector's linearity we apply a nonlinearity correction (as described in Ref. 41) which will scale the ratio $\eta \rightarrow \eta(1+f \mathcal{E}(1-\eta))$ with a factor $f=0.001 \mathrm{GeV}^{-1}$. Limiting the possible change of nonlinearity factor to $10 \%$, from error propagation in Eq. (13), we derive an associated conservative systematic uncertainty of $9.6 \cdot 10^{-4}$ for the asymmetry which is affected negligibly by the correction. 


\section{Gharibyan}

A correlated error could potentially be induced by the detector spatial nonuniformity which affects the Compton edge absolute energy determination in case of Compton and Bremsstrahlung beams vertical separation. ${ }^{41}$ In the asymmetry, however, the non-uniformity is negligible when the Bremsstrahlung from electrons and positrons overlap (Eq. (13) effectively depends on $\eta_{p} / \eta_{e}=\left(C_{\max }^{p} / C_{\max }^{e}\right)$. $\left(B_{\max }^{e} / B_{\max }^{p}\right)$ ratio). This is the usual case with the calorimeter centered on the Compton beam during polarization measurements. Then, the electron and positron Bremsstrahlung vertical overlap is provided by geometrical constraints, flat orbit requirements and the polarimeter setup location at HERA West - away from the routine beam-tuning regions at the lepton-proton interaction points in HERA East, North and South. In order to depart from the assumed $100 \%$ spatial correlation of $e^{-}$and $e^{+}$Bremsstrahlung beams, we allow a $10 \%$ mismatch (correlation coefficient $\left.C_{f}=0.9\right)$ to estimate the detector non-uniformity influence on the measured asymmetry. Thus, the quoted $1 \%$ spatial non-uniformity $\underline{40}$ will result in a $2.2 \cdot 10^{-3}$ correlated systematic error in the asymmetry according to correlated error propagation formula

$$
(\delta A)^{2}=\left(d A_{p}\right)^{2}+\left(d A_{e}\right)^{2}-2 C_{f} d A_{p} d A_{e},
$$

with $d A_{p}=\frac{\partial A}{\partial \eta_{p}} \Delta \eta_{p}, d A_{e}=\frac{\partial A}{\partial \eta_{e}} \Delta \eta_{e}$ and $\Delta \eta_{p} / \eta_{p}=\Delta \eta_{e} / \eta_{e}=0.01$.

Other minor instrumental false asymmetry sources are electronic pedestal offsets, estimated to be $\pm 4 \mathrm{MeV}$ with $\approx 3 \cdot 10^{-4}$ contribution to asymmetry and luminosity-dependent pile-up photons. The latter amounts to 0.02 and 0.0015 photons/bunch for the electrons and positrons, respectively, and, in our case, will tend to reduce any true asymmetry so, it cannot be responsible for the observed effect. An ignorable, about the order of $m$, contribution to electron-positron asymmetry is coming from Bremsstrahlung edge charge dependence. The quoted and calculated errors are listed in Table 1.

There are also potential contributions from chiral sources collected in Table 2. To estimate the magnitudes of these effects, I have explored the HERA transverse polarimeter setup simulation code. .44

Table 1. Errors of the measured asymmetry $A=1.3 \cdot 10^{-2}$.

\begin{tabular}{lcc}
\hline \multicolumn{1}{c}{ Source } & Magnitude (rel.) & Error $\Delta A$ \\
\hline Statistical fluctuations & $1.4 \cdot 10^{-3}$ & $1.3 \cdot 10^{-3}$ \\
Laser frequency shift & $10^{-5}$ & $\approx 0$ \\
Electron mass' error & $3 \cdot 10^{-7}$ & $\approx 0$ \\
Interaction angle drift & $3 \cdot 10^{-6}$ & $\approx 0$ \\
HERA-e energy spread & $10^{-3}$ & $7.9 \cdot 10^{-4}$ \\
Nonlinearity change & $10^{-1}$ & $9.6 \cdot 10^{-4}$ \\
Beams vertical mismatch & $10^{-1}$ & $2.2 \cdot 10^{-3}$ \\
Electronic pedestal shift & $2.9 \cdot 10^{-4}$ & $3 \cdot 10^{-4}$ \\
Pile-up photons & $7.5 \cdot 10^{-2}$ & $\leq 0$ \\
$e^{+}$Bremsstrahlung edge & $7.2 \cdot 10^{-5}$ & $\approx 0$ \\
\hline
\end{tabular}


Table 2. Systematic contributions from chiral sources.

\begin{tabular}{lcc}
\hline Source & Magnitude & Error $\Delta A$ \\
\hline Magnetic field & $50 \mu T$ & $2.3 \cdot 10^{-26}$ \\
Earth's spin & $2 \cdot 10^{-23} \mathrm{eV}$ & $8.0 \cdot 10^{-30}$ \\
Lepton polarization & 0.6 & $3.1 \cdot 10^{-6}$ \\
Laser linear polarization & 0.2 & $5.4 \cdot 10^{-7}$ \\
\hline
\end{tabular}

A tiny effect from the stray magnetic fields have been calculated using Eq. (11). The Earth's rotation gravitomagnetic impact on elementary particles amounts to $\hbar g / c=2.2 \cdot 10^{-23} \mathrm{eV}$, where $g$ is the gravitational acceleration in the laboratory. ${ }^{45}$ This contribution to the measured asymmetry is also negligibly small. Compared to the birefringent magnetic field and Earth's spin, the instrumental chiral effects, responsible for false asymmetries, are larger by many orders of magnitude. The effects are induced by a change of laser polarization either linear or circular, the latter in convolution with the lepton spin. An energy dependence of the induced asymmetries (for $100 \%$ polarized leptons) is plotted in Fig. 4 for HERA polarimeter parameters. Though the mentioned polarized effects cannot directly affect the Compton edge photons to alter the observed asymmetry, an energy smearing and spatial non-uniformity of the detector can mimic a Compton edge shift. Simulations with conservatively high values of the lepton and linear light polarizations show artificial asymmetries below $10^{-5}$ value.

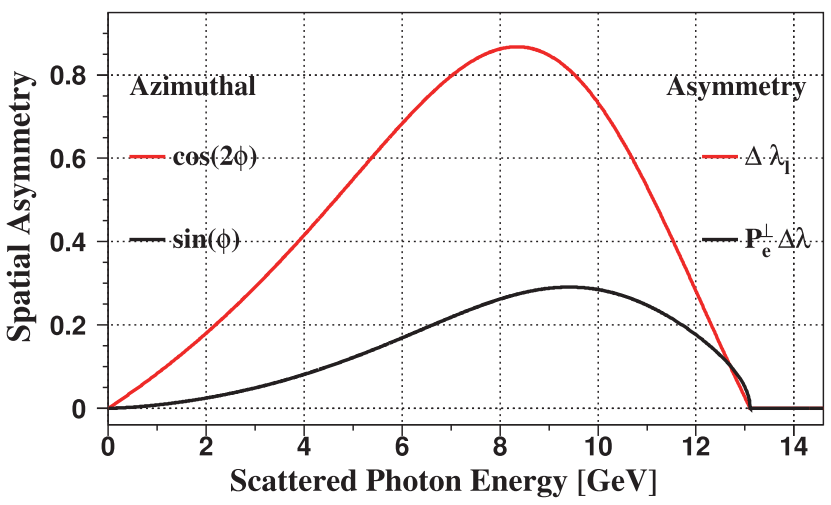

Fig. 4. Asymmetries in Compton scattering associated with the initial photon's polarization (linear $\lambda_{l}$ and circular $\lambda$ ) changes. Analyzing powers (asymmetries for the cases $\left|\Delta \lambda_{l}\right|=1,\left|P_{e} \Delta \lambda\right|=1$ ) of the HERA transverse polarimeter setup.

With a quadratic sum of the estimated systematic and statistical errors, the measured asymmetry and its error become

$$
A_{e p 0-}=(1.293 \pm 0.286) \cdot 10^{-2},
$$




\section{Gharibyan}

which differs from zero by more than $4 \sigma$ confidence. Within an extreme conservative approach, a linear summation of all the errors reduces the deviation to $3.6 \sigma$ confidence interval.

Separate contributions of the gravitational charge and space parity violations cannot be derived from this asymmetry alone. Instead, one can assume space $\mathrm{P}$ parity conservation and calculate maximally possible electron-positron charge $\mathrm{C}$ parity violation by inserting the observed asymmetry into Eq. (8):

$$
\Delta U_{G p}=(-9.0 \pm 2.0) \cdot 10^{-12} .
$$

In a similar way, within a perfect $\mathrm{C}$ parity conservation, one will have maximal $\mathrm{P}$ parity violation magnitude using Eq. (10):

$$
\Delta U_{G-}=(1.28 \pm 0.29) \cdot 10^{-11} .
$$

In a general case of $\mathrm{C} \& \mathrm{P}$ violation, the $\mathrm{C}$ and $\mathrm{P}$ symmetries are violated to lesser degrees. Obtained signs of violations correspond to a stronger gravitational coupling for the left helicity electrons relative to the right helicity positrons.

\section{Conclusions}

Applying a gravitational field-induced refraction and assuming an equivalence principle violation in a general form $\Delta G_{p} / G$ for positrons and $\Delta G_{-} / G$ for helicity, an outstanding sensitivity has been demonstrated for the high-energy Compton scattering to such gravitational anomaly. Within the developed formalism, the HERA Compton polarimeter's recorded spectra with electrons and positrons strongly disfavor the positron's anti-gravity and show a significant deviation of the $\Delta G_{p} / G$ or $\Delta G_{-} / G$ from zero. The last claim is based on a detected 0.013 energy asymmetry, which is a large number compared to the laser and lepton beam energy relative uncertainty of $10^{-5}$ and $10^{-3}$, respectively. The remaining source of a possible systematic energy error is the detector that is greatly eliminated from final result by using the asymmetry instead of absolute energy measurements. However, additional uncorrelated systematic errors may impair the outcome and, claiming a definite observation of CP parity violation at high-energy gravitational interactions would require the following:

- a thorough analysis of many Compton spectra accumulated and recorded by the HERA during its running period;

- elimination of possible electroweak sources that can mimic such result;

- experimental verification at other accelerators.

In the absence of these, the measured electron-positron asymmetry can only be considered as a hint for the gravitational symmetry breaking and an invitation for further studies. New experiments, however, will require future $e^{-} e^{+}$machines with sufficiently high $\gamma$ or a precise setup on the currently running $6 \mathrm{GeV}$ accelerator PETRA-III with the highest positron energy available. Anyway, it is worth the 
efforts since high-energy violation of the equivalence principle and gravitational $\mathrm{CP}$ parity could reveal an interaction to massive or lower spin gravitons.

\section{Acknowledgments}

I thank B. Sobloher and S. Schmitt for providing details about the positron generated spectra, and R. Brinkmann for details about the electron measurement and the HERA. I am also thankful to A. Buniatyan and K. Balewski for useful discussions.

\section{References}

1. A. Einstein, Ann. Phys. 49, 769 (1916); Ann. Phys. 14, 517 (2005).

2. G. Amelino-Camelia, Living Rev. Relativ. 16, 5 (2013).

3. M. M. Nieto and J. T. Goldman, Phys. Rep. 205, 221 (1991).

4. Particle Data Group Collab. (K. A. Olive et al.), Chinese Phys. C 38, 090001 (2014).

5. M. Villata, EPL 94, 20001 (2011).

6. Supernova Search Team Collab. (A. G. Riess et al.), Astron. J. 116, 1009 (1998).

7. Particle Data Group Collab. (J. Beringer et al.), Phys. Rev. D 86, 010001 (2012).

8. A. S. Goldhaber and M. M. Nieto, Rev. Mod. Phys. 82, 939 (2010).

9. A. V. Kostelecky and J. D. Tasson, Phys. Rev. D 83, 016013 (2011).

10. A. Kostelecky and A. J. Vargas, arXiv:1506.01706 [hep-ph].

11. S. Schlamminger, K.-Y. Choi, T. A. Wagner, J. H. Gundlach and E. G. Adelberger, Phys. Rev. Lett. 100, 041101 (2008).

12. E. G. Adelberger, J. H. Gundlach, B. R. Heckel, S. Hoedl and S. Schlamminger, Prog. Part. Nucl. Phys. 62, 102 (2009).

13. D. S. M. Alves, M. Jankowiak and P. Saraswat, arXiv:0907.4110 [hep-ph].

14. R. J. Hughes and M. H. Holzscheiter, Phys. Rev. Lett. 66, 854 (1991).

15. P. Scampoli and J. Storey, Mod. Phys. Lett. A 29, 1430017 (2014).

16. ALPHA Collab. (C. Amole et al.), Nat. Commun. 4, 1785 (2013).

17. ATRAP Collab. (G. Gabrielse et al.), Phys. Rev. Lett. 108, 1133002 (2012).

18. W. T. Ni, Rep. Prog. Phys. 73, 056901 (2010).

19. V. A. Kostelecky, Phys. Rev. D 69, 105009 (2004).

20. F. W. Hehl, P. Von Der Heyde, G. D. Kerlick and J. M. Nester, Rev. Mod. Phys. 48, 393 (1976).

21. J. E. Moody and F. Wilczek, Phys. Rev. D 30, 130 (1984).

22. B. R. Heckel, E. G. Adelberger, C. E. Cramer, T. S. Cook, S. Schlamminger and U. Schmidt, Phys. Rev. D 78, 092006 (2008).

23. V. Gharibyan, Phys. Rev. Lett. 109, 141103 (2012).

24. V. Gharibyan, Accelerator experiments contradicting general relativity, arXiv:1401. 3720 [physics.gen-ph].

25. J. C. Evans, P. M. Alsing, S. Giorgetti and K. K. Nandi, Am. J. Phys. 69, 1103 (2001).

26. F. de Felice, Gen. Relat. Gravit. 2, 347 (1971).

27. A. K. Sen, Astrophysics 53, 560 (2010).

28. J. D. Tasson, Rep. Prog. Phys. 77, 062901 (2014).

29. S. Liberati, Class. Quantum Grav. 30, 133001 (2013).

30. V. A. Kostelecky and N. Russell, Rev. Mod. Phys. 83, 11 (2011).

31. T. Kalaydzhyan, Phys. Lett. B 751, 29 (2015).

32. J.-P. Bocquet et al., Phys. Rev. Lett. 104, 241601 (2010).

33. W. H. McMaster, Rev. Mod. Phys. 33, 8 (1961). 
34. G. L. Kotkin, V. G. Serbo and V. I. Telnov, Phys. Rev. ST Accel. Beams 6, 011001 (2003).

35. F. W. Lipps and H. A. Tolhoek, Physica 20, 85 (1954).

36. J. I. Latorre, P. Pascual and R. Tarrach, Nucl. Phys. B 437, 60 (1995).

37. W. Dittrich and H. Gies, Phys. Rev. D 58, 025004 (1998).

38. L. Knudsen, J. P. Koutchouk, M. Placidi, R. Schmidt, M. Crozon, J. Badier, A. Blondel and B. Dehning, Phys. Lett. B 270, 97 (1991).

39. ALEPH and DELPHI and L3 and OPAL and SLD and LEP Electroweak Working Group and SLD Electroweak Group and SLD Heavy Flavour Group Collab. (S. Schael et al.), Phys. Rep. 427, 257 (2006).

40. D. P. Barber et al., Nucl. Instrum. Meth. A 329, 79 (1993).

41. V. Gharibyan, Phys. Lett. B 611, 231 (2005).

42. F. James and M. Roos, Comput. Phys. Commun. 10, 343 (1975), doi:10.1016/ 0010-4655(75)90039-9.

43. B. Sobloher, R. Fabbri, T. Behnke, J. Olsson, D. Pitzl, S. Schmitt and J. Tomaszewska, Polarisation at HERA - Reanalysis of the HERA II Polarimeter Data -, arXiv:1201. 2894 [physics.ins-det].

44. V. Gharibyan and S. Schmitt, Transverse polarimeter systematic errors, HERMES Internal Report 06-104 (2006), http://www-hermes.desy.de/notes/pub/06-LIB/vahag. 06-104.tpol-syserr.pdf.

45. Y. N. Obukhov, Phys. Rev. Lett. 86, 192 (2001), doi:10.1103/PhysRevLett.86.192. 